\title{
A Rare Low Spin Co(IV) Bis-Silyldiamide with High Thermal Stability: Exploring the Origin of an unusual $S=1 / 2$ configuration
}

\author{
David Zanders, ${ }^{1,2,+^{*}}$ Goran Bačić, ${ }^{2,+}$ Dominique Leckie, ${ }^{3}$ Oluwadamilola O. Odegbesan, ${ }^{2}$ \\ Jeremy Rawson, ${ }^{3}$ Jason D. Masuda, ${ }^{4}$ Anjana Devi ${ }^{1}$ and Seán T. Barry ${ }^{2 *}$ \\ ${ }^{1}$ Inorganic Materials Chemistry, Faculty of Chemistry and Biochemistry, Ruhr University Bochum, Universitätsstraße 150, Bochum, \\ Germany 44780 \\ ${ }^{2}$ Department of Chemistry, Carleton University, 1125 Colonel By Drive, Ottawa, Ontario K1S 5B6, Canada \\ ${ }^{3}$ Department of Chemistry and Biochemistry, University of Windsor, 401 Sunset Avenue, Windsor, Ontario N9B 3P4, Canada \\ ${ }^{4}$ Department of Chemistry, Saint Mary's University, 923 Robie Street, Halifax, Nova Scotia B3H 3C3, Canada \\ Supporting Information Placeholder
}

\begin{abstract}
Attempted preparation of a chelated Co(II) $\beta$-silylamide resulted in the unprecedented disproportionation to $\mathrm{Co}(0)$ and a spirocyclic cobalt(IV) bis $\left.\left.\left(\beta \text {-silyldiamide): [Co[( } \mathrm{N}^{\mathrm{t} B u}\right)_{2} \mathrm{SiMe}_{2}\right]_{2}\right]$ (1). Compound $\mathbf{1}$ exhibits a room temperature magnetic moment of 1.8 B.M and a solid state axial EPR spectrum diagnostic of a rare $S=1 / 2$ configuration. Semicanonical coupled-cluster calculations (DLPNO$\operatorname{CCSD}(\mathrm{T})$ ) revealed the doublet state was clearly preferred $(-27 \mathrm{kcal} / \mathrm{mol})$ over higher spin configurations for which density functional theory (DFT) showed no energetic preference. Unlike other Co(IV) complexes, $\mathbf{1}$ had remarkable thermal stability, and was demonstrated to form a stable self-limiting monolayer in initial atomic layer deposition (ALD) surface saturation tests. The ease of synthesis and high-stability make $\mathbf{1}$ an attractive starting point to begin investigating otherwise inaccessible Co(IV) intermediates and synthesizing new materials.
\end{abstract}

Cobalt complexes are effective and cost-efficient alternatives to Ir- and Rh-based catalysts for a number of important $\mathrm{C}-\mathrm{H}$ bond transformations such as hydroarylation of alkenes, olefins and alkynes, $^{1,2}$ alkynelation of indoles and arenes, ${ }^{3,4}$ as well as alkylation, arylation and borylation of arenes. $^{5,6}$ Recently, high-valent Co(IV) species have also been shown to affect $\mathrm{C}-\mathrm{H}$ bond activation ${ }^{7,8}$ and olefin cyclopropanation. ${ }^{9}$ However, mechanistic investigations of Co(IV) centers are hampered by the lack of isolable Co(IV) complexes. The facile synthesis of robust Co(IV) species has remained a challenge, so their exploitation in the field of catalysis and beyond remains limited.

To date, Co(IV) has been accessed through oneelectron oxidation of $\mathrm{Co}$ (III) complexes with $\mathrm{Br}_{2}$ or $\left[\mathrm{Fe}(\mathrm{III})\left(\mathrm{ClO}_{4}\right)_{3}\right]$ or in the course of cyclic voltammetry. The starting Co(III) complexes typically comprise sterically demanding tetraamido or pyrrolato macrocycles, and the resulting Co(IV) species were unstable above room temperature, exhibiting short lifetimes. In cases where unambiguous Co(IV) species could be isolated, geometries are typically limited to square planar and square pyramidal. ${ }^{10-12}$ Macrocyclic Co(IV) oxo-congeners such as the one introduced by Wang et al. are typically more robust but still suffer from this geometrical restriction. ${ }^{13}$ For $d^{5}$ ions the high spin configuration is more common as it provides the maximum exchange energy and examples of low spin $d^{5}$ are rare. However for Co(IV) the increasing oxidation state affords stronger ligand fields and a finer energetic balance is expected between high spin and low spin electronic configurations. Nevertheless, Co(IV) tetrakis(1norbonyl) [Co(nor) $)_{4}$ provides the only known example of a tetrahedral low spin Co(IV) complex with $S=1 / 2,{ }^{14,15}$ while the more recently reported squashed-tetrahedral Co(IV) tetrakis(ketimide) $\left[\mathrm{Co}\left(\mathrm{N}=\mathrm{C}^{\mathrm{t}} \mathrm{Bu}_{2}\right)_{4}\right]$ exhibits an intermediate spin of $S=$ $3 / 2 \cdot 16$

Silylamines where the $\mathrm{Si}$ is in a $\beta$ position to a bound metal center are excellent ligands to stabilize unusual coordination numbers and oxidation states for elements across the periodic table. ${ }^{17,18}$ The thermal stability and volatility of the well-known three-coordinate $\mathrm{Co}(\mathrm{II})$ complex $\left\{\mathrm{Co}\left[\mathrm{N}\left(\mathrm{SiMe}_{3}\right)_{2}\right]_{2}\right\}_{2}$ prompted us to explore other Co $\beta$-silylamides as potential precursors for vapor phase deposition of Co containing materials. In this paper, we report the serendipitous isolation of $\left[\mathrm{Co}\left[\left(\mathrm{N}^{\mathrm{t} B u}\right)_{2} \mathrm{SiMe}_{2}\right]_{2}\right](\mathbf{1})$, the second nearly tetrahedral Co(IV) complex with a low-spin $S=1 / 2$ ground state configuration and

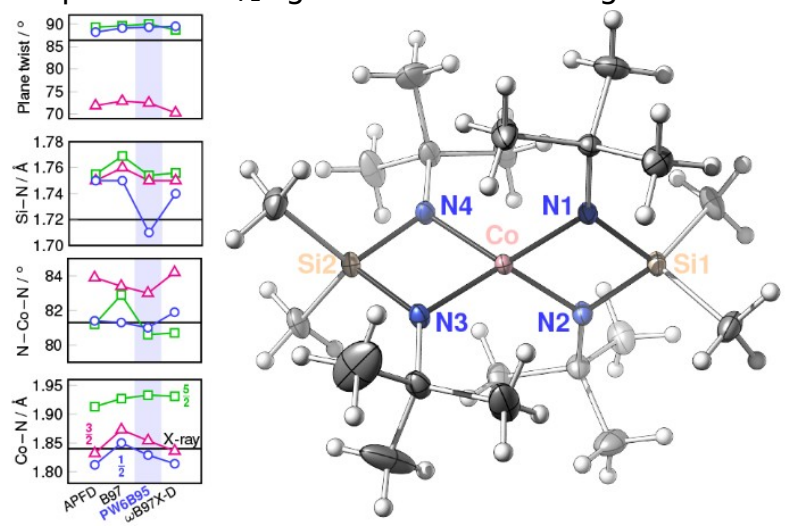


explore its unusual electronic nature through experiment and theory.

Figure 1. Solid-state structure of $\mathbf{1}$ (right) with comparison of DFT optimized geometries from 4 functionals (left) with different ground state configurations: $S=1 / 2$ (blue circles), $S=3 / 2$ (pink triangles) and $S=5 / 2$ (green squares). The (average) crystallographic values are drawn as black lines and the most accurate functional highlighted in blue.

Treatment of suspended $\left[\mathrm{CoCl}_{2}(\mathrm{tmeda})\right]$ with one equivalent of $\left[\left(\mathrm{LiN}^{\mathrm{t} B u}\right)_{2} \mathrm{SiMe}_{2}\right]$ in pentane led to a rapid change in color from ultramarine to dark brown-black. Dark grey particles were observed to precipitate from solution upon stirring following the disproportionation of a presumed $\mathrm{Co}$ (II) intermediate to form $\mathbf{1}$ and metallic $\mathrm{Co}(0)$. Workup of the reaction solution afforded $\mathbf{1}$ as a dark-brown, almost black crystalline solid that was purified either by recrystallization from pentane at $-49{ }^{\circ} \mathrm{C}$ or vacuum sublimation $\left(85^{\circ} \mathrm{C} / 10 \mathrm{mTorr}\right)$. The synthesis scaled well and satisfactory yields (60\% from the ligand) were obtained from $10 \mathrm{mmol}$ batches.

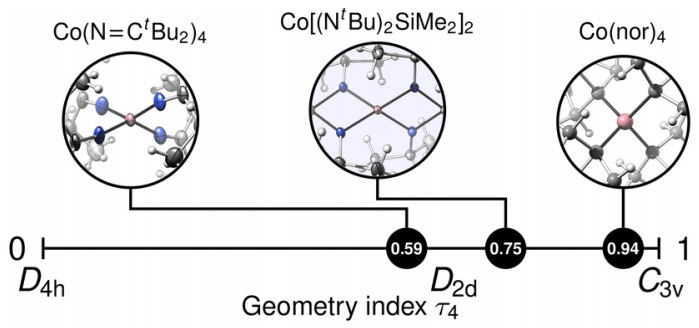

Figure 2. Yang's geometry index $\tau_{4}$ for known Co(IV) complexes.

Compound $\mathbf{1}$. whose solid state structure is depicted in Figure 1 , consists of a distorted tetrahedral inorganic spirocycle of $D_{2 d}$ symmetry. With a Yang geometry index value of $\tau_{4}=0.75,{ }^{19}$ it lies between the two other known four-coordinate homoleptic $\mathrm{Co}(\mathrm{IV})$ complexes: $\left[\mathrm{Co}\left(\mathrm{N}=\mathrm{C}^{\mathrm{t}} \mathrm{Bu}_{2}\right)_{4}\right]\left(\tau_{4}=\right.$ $0.59)^{16}$ and $\left[\mathrm{Co}(\text { nor })_{4}\right]\left(\tau_{4}=0.94\right.$, Figure 2$) .{ }^{15}$ The average $\mathrm{Co}-\mathrm{N}$ bond length of $1.84 \AA$ was only slightly longer than in $\left[\mathrm{Co}\left(\mathrm{N}=\mathrm{C}^{t} \mathrm{Bu}_{2}\right)_{4}\right](1.80 \AA)$, and notably shorter than the $\mathrm{Co}-\mathrm{C}$ bond in $\left[\mathrm{Co}(\text { nor })_{4}\right]$ $(1.92 \AA)$. Coordination around nitrogen in $\mathbf{1}$ was planar, typical of silylamides due to electrostatic repulsion of the polarized $\mathrm{Si}-\mathrm{N}$ bonds. ${ }^{20}$.

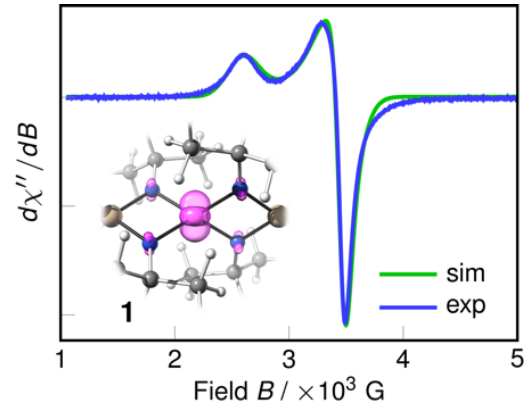

Figure 3. Experimental (blue) and simulated (green) solid state X-band EPR spectra of $\mathbf{1}$. Inset: Alpha spin density of 1 calculated by DFT (def2-QZVPP//PW6B95-D3(BJ)/def2SVP), highlighting the metal-based character of the unpaired electron.

Room temperature magnetic susceptibility measurements on 1 (Gouy method) revealed an effective magnetic moment of $\mu_{\text {eff }}=(1.8 \pm 0.1)$ B.M in the solid state, suggesting $\mathbf{1}$ adopts an unusual low-spin doublet $(S=1 / 2)$ ground state configuration.
This was supported through solid state EPR spectra which displayed axial spectra consistent with a $S=$ $1 / 2$ state $\left(g_{z}=2.6, g_{x}=g_{y}=2.0\right)$ (Figure 3) with gvalues comparable to those computed based on the crystal structure $\left(g_{z}=2.4, g_{x}=g_{y}=2.0\right.$, vide infra). Additional varied temperature (VT) studies from 125 - $300 \mathrm{~K}$ showed no significant change in the EPR signal across this temperature range (Figure $S 7$ ). The pattern of $g$-values is similar to a low spin fourcoordinate $\mathrm{d}^{5} \mathrm{Fe}(\mathrm{III})$ complex reported by Peters which adopted $\mathrm{C}_{3 \mathrm{v}}$ symmetry $\left(g_{z}=2.9, g_{x}=2.0, g_{y}=\right.$ 2.0)..$^{21}$

We used density functional theory (DFT) to understand the origins of the low-spin ground states of 1 and $\left[\mathrm{Co}(\text { nor })_{4}\right]$. We chose four different functionals because of their excellent thermochemical performance, ability to correctly assign spin-states of transition metal complexes, inclusion of dispersion corrections, and their prevalence in the literature: APFD, B97-D3(BJ), PW6B95-D3(BJ) and $\omega B$ 97X-D2. ${ }^{22,23}$ Each of these reproduced the stability of the doublet state of [Co(nor) ${ }_{4}$ ] relative to $S=5 / 2$ and $S=3 / 2$ (ca. -30 and $-60 \mathrm{kcal} / \mathrm{mol},{ }^{24,25}$ respectively). On the other hand, basis set convergence for $\mathbf{1}$ was slow, and the most stable spin-state remained ambiguous. Agreement among functionals was eventually achieved with a quadruple- $\zeta$ quality basis set (def2-QZVPP) on all atoms, but the possible spin-states of $\mathbf{1}$ were predicted to be nominally equal to the doublet state in energy $\left(\Delta \mathrm{E}\left({ }^{3} / 2\right)=-3.7 \mathrm{kcal} / \mathrm{mol}\right.$ and $\Delta \mathrm{E}\left({ }^{5} / 2\right)=-8.4$ $\mathrm{kcal} / \mathrm{mol}$ on average ). The ambiguous energetics of 1 were in stark contrast to the excellent agreement with experimental geometric and spectroscopic properties in favor of the low-spin configuration (see Figure 1). Here, all functionals showed that the closest geometric agreement to the crystallographically determined structure was with the doublet state. Conversely, metal-ligand bond lengths were elongated in the $S=5 / 2$ geometries, along with poorer agreement among the functionals and with the experimental structure. The predicted $S=3 / 2$ configuration displayed non-perpendicular spirocyclic rings $\left(\mathrm{ca} .72^{\circ}\right)$, providing poor agreement with the X-ray structure. Despite the equal predicted energies, the calculated $g$-tensors (def2QZVPP//PW6B95-D3(BJ)/def2-SVP) for the $S=1 / 2$ state $\left(g_{z}=2.4, g_{x}=g_{y}=2.0\right)$ agreed very well with the solid-state EPR spectrum whereas the $S=5 / 2$ and $\mathrm{S}=3 / 2$ configurations provided poor agreement with the experimental data $\left(g_{z}=g_{x}=g_{y}=2.0\right)$.

Accurate prediction of spin-states with DFT remains challenging due to the inexact exchangecorrelation functional and the description of $\mathbf{1}$ was no exception. Thus, we performed domain-based local pair natural orbital coupled-cluster (DLPNOCCSD(T)/def2-TZVPP) calculations with our best DFToptimized structures (PW6B95-D3(BJ)/def2-SVP) to provide a reference value. This recent formulation provides comparable performance to canonical $\operatorname{CCSD}(\mathrm{T})$, the "gold standard" of quantum chemistry, but with computational cost comparable to DFT. ${ }^{26}$ Indeed, the $a b$ initio calculations show the $S=1 / 2$ state to be $27 \mathrm{kcal} / \mathrm{mol}$ lower in energy than $S=5 / 2$ or $S=3 / 2$. However, since CC provides no orbitals to 
perform analyses with, we decided to rely on our DFT results for the rest of the investigation.

Revisiting solid state structural features, the short Co-Si distance in $\mathbf{1}(2.63 \AA)$ caught our attention as it raises the question whether a $\mathrm{Co}-\mathrm{Si}$ bond exists considering the sum of their covalent radii $(2.25 \AA)$. Naturally, the answer depends on which of the numerous definitions of "chemical bond" is chosen. Mayer bond order (MBO) is a popular and powerful tool to investigate bonding, but for 1 it indicated a Co-Si bond of order $\sim 0.6$ and also predicted $\mathrm{Co}-\mathrm{C}$ bonds to the tert-butyl moieties of order $\sim 0.3$. However, neither the canonical nor localized molecular orbitals showed any obvious bonding orbitals between Co and Si nor $C$, which suggested the "bonds" do not exist.
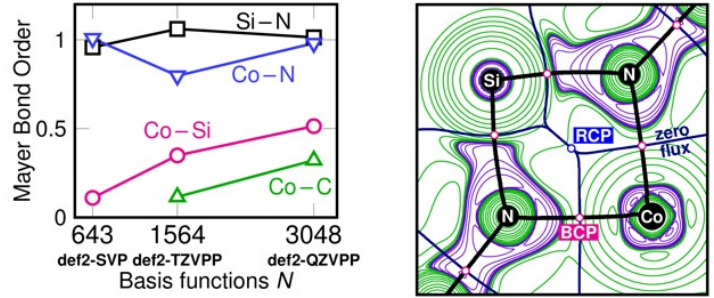

igure 4. Mayer bond orders (MBOs) as a function of number of basis functions $N$ (left), and contour map of $\nabla^{2} \rho$ showing bond critical points (BCPs), the ring critical point (RCP), and the interatomic basins within one heterocycle of 1 (right).

The erroneous assignment of bonds by MBO are likely due to its use of the overlap matrix $S$ considering the proximity of the $\mathrm{Co}, \mathrm{Si}$ and $\mathrm{C}$ atoms. Indeed, there was a positive correlation between the number of basis functions $N$ and the calculated MBOs of Co with $\mathrm{C}$ and Si (Figure 4). Bader's atomsin-molecules (AIM) theory provides a clear, physically justified definition of the chemical bond as a saddlepoint in the topology of the electron density. There were no $(+3,-1)$ bond critical points (BCPs) between $\mathrm{Co}$ and $\mathrm{Si}$ nor $\mathrm{C}$, nor was there any significant charge accumulation between them. This was further supported by Laplacian bond order (LBO) calculations (see SI). In conclusion, there were no Co-Si nor Co-C bonds in $\mathbf{1}$.

Dispersion interactions were recently shown to significantly stabilize $\left[\mathrm{Co}(\text { nor })_{4}\right]_{1}{ }^{24,25}$ and we suspected they played an important role in the structure of $\mathbf{1}$. Johnson et al. described a method to visualize non-covalent interactions ( $\mathrm{NCls)}$ in real space, showing the attractive and repulsive interactions occurring within and between molecules. ${ }^{27} \mathrm{NCl}$ analysis of [Co(nor) $)_{4}$ ] and $\mathbf{1}$ (Figure 5) revealed continuous garlands of attraction between their ligands, evidently directing the geometry and thus electronic nature of the complexes. The attractive surface in $\mathbf{1}$ shows strong attraction between the alkyl groups on one ligand with the other, while the repulsive surface shows steric hinderance only between the outer alkyl groups and the inner inorganic spirocycle. Our assertion that the steric of the ligand force $\mathbf{1}$ into the low spin configuration were supported by further DFT calculations on analogues of $\mathbf{1}$ with smaller $\mathrm{N}$ substituents (i.e., from methyl to iso-propyl): The $S$ $=1 / 2$ state became gradually more favorable with increasing steric bulk (See SI). However, the crossover point could not be quantified due to the shortcomings to describe this system with DFT.

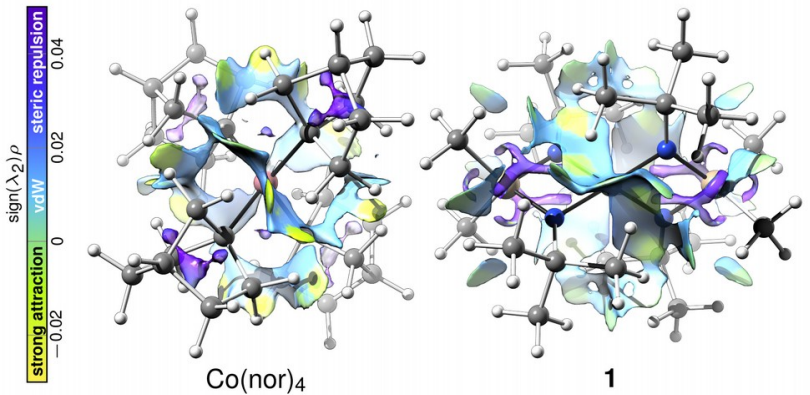

Figure 5. Non-covalent interactions ( $\mathrm{NCls)}$ at work in [Co(nor) $\left.{ }_{4}\right]$ (left) and $\mathbf{1}$ (right). The surfaces are at an isovalue of 0.5 a.u. and colored by the value of $\operatorname{sign}\left(\lambda_{2}\right) \rho$ from -0.03 (yellow/green, attractive), through ca. zero (cyan, van der Waals interaction) to 0.05 a.u. (indigo/purple, steric repulsion).

Our computations to understand the electronic configuration and spin state of $\mathbf{1}$ were complemented by probing its suitability for vapor phase deposition. Thermogravimetric analysis (TGA) and differential thermal calorimetry (DSC) were employed for quantitative assessment of volatility and thermal stability. When a $10.1 \mathrm{mg}$ sample was heated with a linearly increasing temperature ramp, a clean single-step mass loss was observed between $150-230{ }^{\circ} \mathrm{C}$ leaving only a small residual mass of $3.2 \%$ behind (Figure 6a). DSC revealed the melting point at $143{ }^{\circ} \mathrm{C}$ and the onset of decomposition (defined as $5 \%$ of the maximum of the first exothermic event) ${ }^{28}$ to be $197^{\circ} \mathrm{C}$. The "thermal range" between when a compound achieves a vapor pressure of 1 Torr and its decomposition point is often a practical benchmark of its eligibility as a precursor for atomic layer deposition (ALD). TGA can be used to efficiently estimate vapor pressure, and the 1 Torr temperature of spirocyclic $\mathbf{1}$ was estimated to be $(150.4 \pm 0.1){ }^{\circ} \mathrm{C}$ (Figure 6b)..$^{29}$ With a "thermal range" spanning roughly $47^{\circ} \mathrm{C}$, compound $\mathbf{1}$ represents a promising candidate for ALD. To the best of our knowledge, such thermal features are unprecedented among Co(IV) complexes; other potentially volatile species like $\left[\mathrm{Co}(\text { nor })_{4}\right]$ readily decompose upon heating to temperatures of $100{ }^{\circ} \mathrm{C}$ and cannot be sublimed. ${ }^{17}$

The most important characteristic of ALD precursors is the ability to form a stable self-limiting monolayer on a surface. ${ }^{30}$ After all reactive surface sites have undergone a reaction with precursor molecules, no further adsorption should occur, allowing conformal and uniform deposition to take place. ${ }^{31}$ Employing a quartz crystal microbalance (QCM) in our home-built ALD tool, we investigated the potential for $\mathbf{1}$ to fulfill this basic yet fundamental requirement. While the precursor was heated to $135^{\circ} \mathrm{C}$ for delivery, the QCM crystal was heated to $150{ }^{\circ} \mathrm{C}$ for saturation experiments. Compound $\mathbf{1}$ demonstrates self-limiting adsorption on alumina with a mass gain of $(49.2 \pm 0.3) \mathrm{ng} \mathrm{cm}$ over an extended time span of roughly 35 minutes (Figure $6 c$ ). This initial result was encouraging and motivated us to begin ALD process development with $\mathbf{1}$ in an ongoing study. 

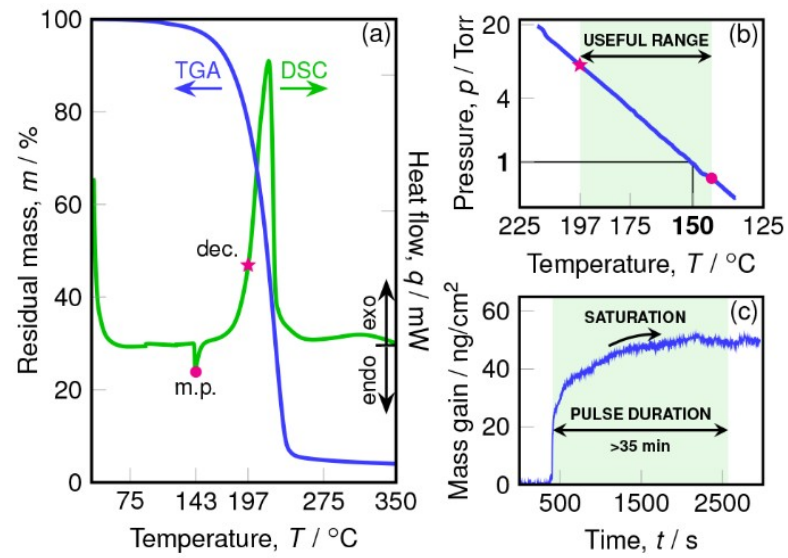

igure 6. a) Thermogravimetric analysis of a $10.1 \mathrm{mg}$ sample (blue line) and differential scanning calorimetry of a $0.3 \mathrm{mg}$ sample (green line) of $\mathbf{1}$ in a temperature range of $40{ }^{\circ} \mathrm{C}$ - $350{ }^{\circ} \mathrm{C}$. b) Vapor pressure - temperature correlation for 1. c) Exposure of an alumina coated quartz crystal microbalance (QCM) substrate to a long pulse of $\mathbf{1}$ demonstrating saturating and self-limited adsorption visualized by the calculated mass gain $\left(\mathrm{ng} \mathrm{cm}^{-2}\right)$. Experimental details are provided in the SI for clarity.

In summary, a thermally stable and volatile homoleptic Co(IV) spirocyclic compound was synthesized in a facile one-step procedure exploiting Co(II) disproportionation. A combination of experimental and computational studies confirmed that it exhibits an unusual low-spin $d^{5}$ configuration, with important similarities and differences to its closest relative $\left[\mathrm{Co}(\text { nor })_{4}\right]$. The remarkable stability of 1 makes it a promising precursor for vapor deposition, and beyond this it represents a simple and appealing starting point for novel high-valent Co(IV) chemistry to be explored.

\section{ASSOCIATED CONTENT}

\section{Supporting Information}

The Supporting Information is available free of charge on the ACS Publications website.

Synthetic procedures, PXRD data, TGA and DSC data, Additional X-ray crystallographic details, ALD saturation experimental, NMR spectra, IR data, UVVIS spectra, EI-MS data, EPR data, Computational details, Cartesian geometries of calculated structures (PDF)

\section{AUTHOR INFORMATION}

\section{Corresponding Authors}

*david.zanders@rub.de

*sean_barry@carleton.ca

\section{Author Contributions}

$\dagger$ David Zanders and Goran Bačic contributed equally to this work.

\section{Notes}

The authors declare no competing financial interests. All authors have given approval to the final version of the manuscript.

\section{ACKNOWLEDGMENT}

We thank Sharon Curtis at University of Ottawa for supporting this work with mass spectrometry, and G.B. thanks Charles L.B. MacDonald and Erin R. Johnson for fruitful discussions. The authors from RUB thank the DFG (SFB-TR87) for financial support. D.Z. wishes to thank the German Academic Exchange Service (DAAD-1-year fellow program) and the Fond of Chemical Industries (Kekulé fellowship) for ideational and financial support. G.B. and S.T.B acknowledge the QEII-OGSST program for funding and Carleton University for support. S.T.B. acknowledges the Natural Sciences and Engineering Research Council of Canada (NSERC, RGPIN-201906213).

REFERENCESX(1) Lee, P.-S.; Fujita, T.; Yoshikai, N. Cobalt-catalyzed, room-temperature addition of aromatic imines to alkynes via directed $\mathrm{C}-\mathrm{H}$ bond activation. Journal of the American Chemical Society 2011, 133, 17283-17295.

(2) Gao, K.; Yoshikai, N. Cobalt-phenanthroline catalysts for the ortho alkylation of aromatic imines under mild reaction conditions. Angewandte Chemie (International ed. in English) 2011, 50, 6888-6892.

(3) Moselage, M.; Sauermann, N.; Richter, S. C.; Ackermann, L. C-H alkenylations with alkenyl acetates, phosphates, carbonates, and carbamates by cobalt catalysis at $23^{\circ} \mathrm{C}$. Angewandte Chemie (International ed. in English) 2015, 54, 6352-6355.

(4) Song, W.; Ackermann, L. Cobalt-catalyzed direct arylation and benzylation by $\mathrm{C}-\mathrm{H} / \mathrm{C}-\mathrm{O}$ cleavage with sulfamates, carbamates, and phosphates. Angewandte Chemie (International ed. in English) 2012, 51, 82518254.

(5) Obligacion, J. V.; Semproni, S. P.; Chirik, P. J. Cobaltcatalyzed C-H borylation. Journal of the American Chemical Society 2014, 136, 4133-4136.

(6) Gao, K.; Yoshikai, N. Cobalt-catalyzed ortho alkylation of aromatic imines with primary and secondary alkyl halides. Journal of the American Chemical Society 2013, 135, 9279-9282.

(7) Landge, V. G.; Jaiswal, G.; Balaraman, E. CobaltCatalyzed Bis-alkynylation of Amides via Double $\mathrm{C}-\mathrm{H}$ Bond Activation. Organic letters 2016, 18, 812-815.

(8) Zhang, L.; Liu, Y.; Deng, L. Three-Coordinate Cobalt(IV) and Cobalt(V) Imido Complexes with NHeterocyclic Carbene Ligation: Synthesis, Structure, and Their Distinct Reactivity in $\mathrm{C}-\mathrm{H}$ Bond Amination. J. Am. Chem. Soc. 2014, 136, 15525-15528.

(9) Bellow, J. A.; Stoian, S. A.; van Tol, J.; Ozarowski, A.; Lord, R. L.; Groysman, S. Synthesis and Characterization of a Stable High-Valent Cobalt Carbene Complex. Journal of the American Chemical Society 2016, 138, 5531-5534.

(10) Collins, T. J.; Powell, R. D.; Slebodnick, C.; Uffelman, E. S. Stable highly oxidizing cobalt complexes of macrocyclic ligands. J. Am. Chem. Soc. 1991, 113, 8419-8425.

(11) Topich, J.; Halpern, J. Organobis(dioximato)cobalt(IV) complexes: Electron paramagnetic resonance spectra and electronic structures. Inorg. Chem. 1979, 18, 1339-1343.

(12) Will, S.; Lex, J.; Vogel, E.; Adamian, V. A.; van Caemelbecke, E.; Kadish, K. M. Synthesis, Characterization, and Electrochemistry of $\sigma$-Bonded Cobalt Corroles in High Oxidation States. Inorg. Chem. 1996, 35, 5577-5583.

(13) Wang, B.; Lee, Y.-M.; Tcho, W.-Y.; Tussupbayev, S.; Kim, S.-T.; Kim, Y.; Seo, M. S.; Cho, K.-B.; Dede, Y.; Keegan, B. C. et al. Synthesis and reactivity of a 
mononuclear non-haem cobalt(IV)-oxo complex. Nature communications 2017, 8, 14839.

(14) Byrne, E. K.; Richeson, D. S.; Theopold, K. H. Tetrakis(1-norbornyl)cobalt, a low spin tetrahedral complex of a first row transition metal. J. Chem. Soc., Chem. Commun. 1986, 1491.

(15) Bower, B. K.; Tennent, H. G. Transition metal bicyclo[2.2.1] hept-1-yls. J. Am. Chem. Soc. 1972, 94, 2512-2514.

(16) Lewis, R. A.; George, S. P.; Chapovetsky, A.; Wu, G.; Figueroa, J. S.; Hayton, T. W. Synthesis of a cobalt(IV) ketimide with a squashed tetrahedral geometry. Chemical communications (Cambridge, England) 2013, 49, 2888-2890.

(17) Veith, M. Cyclische Stickstoffderivat des vier- und zweiwertigen Zinns. Angew. Chem. 1975, 87, 287-288.

(18) Lappert, M. F.; Protchenko, A.; Power, P.; Seeber, A. Metal amide chemistry; Wiley: Chichester, 2009.

(19) Yang, L.; Powell, D. R.; Houser, R. P. Structural variation in copper(I) complexes with

pyridylmethylamide ligands: structural analysis with a new four-coordinate geometry index, tau4. Dalton transactions (Cambridge, England : 2003) 2007, 955964.

(20) Mo, Y.; Zhang, Y.; Gao, J. A Simple Electrostatic Model for Trisilylamine: Theoretical Examinations of the $n \rightarrow \sigma^{*}$ Negative Hyperconjugation, $p \pi \rightarrow d \pi$ Bonding, and Stereoelectronic Interaction. J. Am. Chem. Soc. 1999, 121, 5737-5742.

(21) Brown, S. D.; Betley, T. A.; Peters, J. C. A low-spin d5 iron imide: nitrene capture by low-coordinate iron(I) provides the 4-coordinate Fe(III) complex $\mathrm{PhB}(\mathrm{CH} 2 \mathrm{PPh} 2) 3 \mathrm{Fe}=\mathrm{N}$-p-tolyl. Journal of the American Chemical Society 2003, 125, 322-323.

(22) Verma, P.; Varga, Z.; Klein, J. E. M. N.; Cramer, C. J.; Que, L.; Truhlar, D. G. Assessment of electronic structure methods for the determination of the ground spin states of $\mathrm{Fe}(\mathrm{ii}), \mathrm{Fe}(\mathrm{iii})$ and $\mathrm{Fe}(\mathrm{iv})$ complexes. Physical chemistry chemical physics : PCCP 2017, 19, 13049-13069.

(23) Goerigk, L.; Hansen, A.; Bauer, C.; Ehrlich, S.; Najibi, A.; Grimme, S. A look at the density functional theory zoo with the advanced GMTKN55 database for general main group thermochemistry, kinetics and noncovalent interactions. Physical chemistry chemical physics : PCCP 2017, 19, 32184-32215.

(24) Li, H.; Hu, Y.; Di Wan; Zhang, Z.; Fan, Q.; King, R. B.; Schaefer, H. F. Dispersion Effects in Stabilizing Organometallic Compounds: Tetra-1-norbornyl Derivatives of the First-Row Transition Metals as Exceptional Examples. The journal of physical chemistry. A 2019, 123, 9514-9519.

(25) Liptrot, D. J.; Guo, J.-D.; Nagase, S.; Power, P. P. Dispersion Forces, Disproportionation, and Stable HighValent Late Transition Metal Alkyls. Angew. Chem. 2016, 128, 14986-14989.

(26) Riplinger, C.; Sandhoefer, B.; Hansen, A.; Neese, F. Natural triple excitations in local coupled cluster calculations with pair natural orbitals. The Journal of Chemical Physics 2013, 139, 134101.

(27) Johnson, E. R.; Keinan, S.; Mori-Sánchez, P.; Contreras-García, J.; Cohen, A. J.; Yang, W. Revealing noncovalent interactions. Journal of the American Chemical Society 2010, 132, 6498-6506.

(28) Griffiths, M. B. E.; Dubrawski, Z. S.; Bačić, G.; Japahuge, A.; Masuda, J. D.; Zeng, T.; Barry, S. T. Controlling the Thermal Stability and Volatility of Organogold(I) Compounds for Vapor Deposition with Complementary Ligand Design. Eur. J. Inorg. Chem. 2019, 2019, 4927-4938.

(29) Kunte, G. V.; Shivashankar, S. A.; Umarji, A. M. Thermogravimetric evaluation of the suitability of precursors for MOCVD. Meas. Sci. Technol. 2008, 19, 25704.

(30) George, S. M. Atomic layer deposition: an overview. Chemical reviews 2010, 110, 111-131.

(31) Leskelä, M.; Ritala, M. Atomic layer deposition (ALD): From precursors to thin film structures. Thin Solid Films 2002, 409, 138-146. 
